\title{
Can Mindfulness-Based Interventions Benefit People with Dementia? Drawing on The Evidence from a Systematic Review in Populations with Cognitive Impairments
}

Joanne Chan , Dara K. Y. Leung, Holly Walton, Gloria H. Y. Wong \& Aimee Spector

To cite this article: Joanne Chan , Dara K. Y. Leung , Holly Walton, Gloria H. Y. Wong \& Aimee Spector (2020): Can Mindfulness-Based Interventions Benefit People with Dementia? Drawing on The Evidence from a Systematic Review in Populations with Cognitive Impairments, Expert Review of Neurotherapeutics, DOI: 10.1080/14737175.2020.1810571

To link to this article: https://doi.org/10.1080/14737175.2020.1810571

Accepted author version posted online: 26

Aug 2020.

Submit your article to this journal $₫$

Џ Article views: 2

Q View related articles $\asymp$

View Crossmark data $₫$ 
Publisher: Taylor \& Francis \& Informa UK Limited, trading as Taylor \& Francis Group

Journal: Expert Review of Neurotherapeutics

DOI: $10.1080 / 14737175.2020 .1810571$

Can Mindfulness-Based Interventions Benefit People with

Dementia? Drawing on The Evidence from a Systematic Review in Populations with Cognitive Impairments

Joanne Chan ${ }^{1 \#}$, Dara K. Y. Leung ${ }^{2 \#}$, Holly Walton ${ }^{1}$, Gloria H. Y. Wong ${ }^{2}$, \& Aimee Spector $^{1^{*}}$

1. Department of Clinical, Educational and Health Psychology, University College London, London, United Kingdom

2. Department of Social Work and Social Administration, The University of Hong Kong, Hong Kong SAR

\#These authors contributed equally to this article

${ }^{*}$ Corresponding author:

Aimee Spector

Address: University College London, Department of Clinical, Educational and Health Psychology, $4^{\text {th }}$ Floor, 1-19 Torrington Place, London, WC1E 7HB, London, UK, Telephone: 02076791844

Email: a.spector@ucl.ac.uk

\section{Abstract}


Introduction: Non-pharmacological interventions that promote quality of life in people with dementia are urgently needed. To accelerate development, evidencebased psychotherapies used in other populations can be considered. Mindfulnessbased interventions with standardised protocols, namely mindfulness-based cognitive therapy (MBCT) and mindfulness-based stress reduction (MBSR), may be effective in people with dementia, although tailoring for cognitive impairment may be needed. Evidence from other cognitive disorders can inform research.

Areas covered: The authors reviewed 12 studies of MBCT or MBSR conducted in people with cognitive impairments, including ten in stroke, traumatic brain injury, and mild cognitive impairment; and two in dementia. Protocol modifications, outcomes, and evidence quality were analysed. Common themes to address cognitive difficulties included: shortened session duration, use of memory aids, increase in repetition, simplified language, and omitted retreat sessions.

Expert opinion: MBCT and MBSR can be applied without drastic modifications in people with cognitive impairment. Their effectiveness in people with dementia remains unknown: empirical studies using/adapting evidence based MBCT/MBSR protocols in this population is seriously lacking. Studies used a diverse range of outcome measures, which made direct comparison difficult. Further research with high methodological quality, sufficient power and longer follow-up are urgently needed. Development of manuals would enhance the replicability of future studies.

Keywords: dementia, mild cognitive impairment, mindfulness-based cognitive therapy, mindfulness-based stress reduction, intervention modification

\section{Article highlights}


- Both mindfulness-based cognitive therapy (MBCT) and mindfulness-based stress reduction (MBSR) appear feasible in people with cognitive impairments

- Minor modifications were mainly observed in delivery format such as duration, without having to tailor the intervention content according to cognitive ability such as abstract thinking.

- Anecdotal evidence suggests mindfulness-based intervention may be effective even in later stages of dementia.

- Empirical studies of mindfulness-based intervention using/adapting evidencebased protocols of MBCT or MBSR in people with dementia is seriously lacking.

- Future studies need to also include measures of mindfulness to inform the theoretical basis of applying mindfulness-based interventions in people living with dementia.

\section{Introduction}

\subsection{Non-pharmacological interventions in dementia}

To support the 50 million people with dementia globally [1], identifying a cure or a disease-modifying therapy by 2025 has been set as a priority [2]. However, due to the intrinsic difficulties in dementia drug research [3], finding a cure remains an ambitious goal, and we must take into consideration the possibility that most people with dementia would not live long enough to benefit from the development of an eventual cure. Efforts are needed in parallel to identify ways to maintain or improve the quality of life in people with dementia [4].

Apart from medication treatment using acetylcholinesterase inhibitors (AChEls; e.g. Donepezil) to maintain cognitive function or delay cognitive decline 
with in people with mild to moderate dementia [5], non-pharmacological interventions that enhance cognition, reduce behavioural and psychological symptoms of dementia (BPSD), and promote psychosocial wellbeing including wellbeing and quality of life are urgently needed. To-date, only a few non-pharmacological interventions have accumulated enough evidence to support their routine use in clinical services and long-term care settings, such as Cognitive Stimulation Therapy (CST). In the case of CST, the key trial demonstrating its clinical benefit (with an effect size on cognitive outcome similar to drug treatment with improved quality of life) was published back in 2003 [6]. Wider implementation of CST came later, for example it was not until 2011 when the Alzheimer's Disease International stated that it "should routinely be given to people with early stage dementia" [5], and similarly by the UK Social Care Institute for Excellence (SCIE) and National Institute of Health and Clinical Excellence (NICE) [7], based on a review of evidence. Two metaanalyses have since been published, providing further evidence on the effects of CST in delaying cognitive decline and improving quality of life $[8,9]$. In the more recent update of the NICE guidelines, CST and activities tailored to the person's preferences remain the only two recommended strategies to promote cognition, independence, and wellbeing in people with dementia [10]. Available options of evidence-based, non-pharmacological interventions are still limited.

Many promising candidates of non-pharmacological interventions are in the pipeline. For example, the 2018 NICE guidelines suggested that group reminiscence therapy and cognitive rehabilitation can be considered [10]. For reminiscence therapy, in an earlier Cochrane review in 2000 , only two trials could be identified, although some possible benefits were noted, and directions for future research 
highlighted [11]. An update in 2005 identified four trials, when results were still inconclusive, but several promising indications were noted, such as designs that incorporate relationship-centred care [12]. By 2018, the most updated review suggested that there seem to be some benefits in quality of life, cognition, communication, and mood [13]. For cognitive rehabilitation, a Cochrane review is currently underway [14]. Interventions targeting behavioural, psychological, and psychosocial outcomes, such as the Well-being and Health for people with Dementia (WHELD) programme, have shown evidence of improving agitation and quality of life [15]; earlier studies have also shown lower agitation in residential care homes providing dementia care mapping or person-centred care [16]. However, interpreting results from these complex interventions are often not straightforward; for example, a similar programme targeting agitation and quality of life in residential care units, Managing Agitation and Raising Quality of Life (MARQUE), found benefits in qualityadjusted life years gain but not in managing agitation [17]. Similarly, it remains inclusive whether dementia care management is efficacious, due to protocol and research methodological differences [18]. The development of these interventions from concepts to evidence and large-scale implementation often takes decades (for a recent brief summary of these non-pharmacological interviews in their costeffectiveness, which is related to scalability, see [19]).

\subsection{Evidence-based psychological therapies in other populations}

The above interventions are in general interventions specific to dementia, mostly with a focus on cognition. For other needs that people with dementia share with other populations, such as mood and anxiety symptoms, the potential of applying/adapting evidence-based psychological treatments is under-explored. For 
example, there is some evidence that cognitive behavioural therapy, counselling, and interpersonal psychodynamic therapy can reduce depressive symptoms and clinician-rated anxiety symptoms in people with dementia, although more studies are needed [20]. Notably, newer intervention approaches such as mindfulness-based interventions, sometimes referred to as the 'third wave' of psychological treatment [21], with an emphasis on issues such as mindfulness, values, and acceptance, are theoretically applicable in dementia.

Among these newer approaches, research evidence is more readily available for evaluating mindfulness-based interventions. The concept of 'mindfulness' is a way of paying attention on purpose, in the present moment and in a non-judgmental manner [22]. Mindfulness-based interventions, such as mindfulness-based stress reduction (MBSR) [22] and mindfulness-based cognitive therapy (MBCT) [23], involve both focused attention and open monitoring training [24]. Mindfulness-based stress reduction commonly involves eight weekly 2.5-hour group sessions with daily 45-minute home practice, and a retreat day of six hours between weeks 6 and 7 [22]. Mindfulness-based cognitive therapy consists of eight, weekly 2-hour sessions which incorporate both meditation techniques from the MBSR program and elements of cognitive behavioural therapy. It aims to develop participants' meta-cognitive awareness by focusing on greater awareness of their relationship to their thoughts and feelings, without challenging specific thoughts [25].

Several reviews have advocated mindfulness-based interventions as a promising intervention for treating anxiety and mood problems in clinical populations [26]. Reviews and meta-analyses suggest that a MBCT is effective for preventing 
relapse in people with recurrent major depressive disorder in remission [25, 27-29] and it is recommended by the UK NICE guidelines [30] for depression relapse prevention. On the other hand, MBSR is showing initial evidence of effectiveness in improving psychological functioning in physical health conditions, such as cancer, fibromyalgia, chronic pain, multiple sclerosis, psoriasis, HIV and chronic obstructive lung disease, and also in healthy participants [29, 31-33]. In people with acquired chronic cognitive impairment, such as dementia, given their effects on cognitive capability that these mindfulness-based interventions assume, whether these psychotherapies (especially those requiring higher cognitive functions such as metacognition) are feasible and effective remains an empirical question.

Research on mindfulness-based interventions in populations with cognitive impairment is emerging. Cognitive impairment describes a decline in cognition compared to premorbid levels in domains such as memory, language, attention/executive functioning, and/or visuospatial skills. It is common following brain injury and is a feature of neurodegenerative disorders such as dementia. They are considered distinct from lifelong cognitive impairment such as intellectual disabilities, which refer to a significant impairment of intelligence and social functioning with onset before adulthood [34]. The major types of acquired cognitive impairment include acquired brain injury, mild cognitive impairment (MCI), and dementia. Acquired brain injury can include traumatic brain injury, vascular accidents, cerebral anoxia, brain infection and toxic or metabolic insult. The diagnostic criteria for $\mathrm{MCl}$ include: subjective reports of cognitive changes by the affected individual or observers; objective impairment in one or more cognitive domains; independence in functional abilities with minimal aids/assistance; and 
absence of dementia [35]. It could be the result of different etiologies, including Alzheimer's disease, vascular cognitive impairment, frontotemporal dementia, dementia with Lewy bodies, and depression [36]. Although people with $\mathrm{MCl}$ may remain stable, return to normal, or progress to dementia depending on etiology and other factors, they are in general at a higher risk of dementia compared with those who never had $\mathrm{MCl}$, with updated clinical guidelines suggesting $55 \%$ to $65 \%$ ultimately converting to dementia [37].

In an earlier review on mindfulness-based interventions in people with transient ischemic attack and stroke [38], despite the limited number of studies included and methodological limitations in these studies, there was an indication of short-term benefits in depression, anxiety, mental fatigue, and quality of life, and no indication of adverse events. A meta-analysis of four studies found a moderate effect size in mindfulness-based interventions for reducing fatigue in people with neurological conditions or acquired brain injuries [39], although the studies did not focus on cognitive impairment that may be associated.

\subsection{Objectives}

In this review, we first examined more broadly the application of mindfulness-based interventions in people with cognitive impairments other than dementia that are chronic and non-developmental in nature, including stroke, traumatic brain injury, and $\mathrm{MCl}$, which can have wide ranging impact on various cognitive domains such as memory, language, attention, executive functioning, and visuospatial skills. Due to the wide range of interventions that can be termed mindfulness-based interventions, and for clarity of concept, we are focusing our review on studies on MBCT or MBSR, or a modification of these programmes. We examined particularly how the protocols 
were modified for cognitive impairment and what outcome measures used, to identify implications for mindfulness-based interventions for people with dementia. Based on this general overview, we then critically reviewed specific evidence on mindfulnessbased intervention in people with dementia, in regard to the intervention protocol modifications, outcomes, and evidence quality. This review aims to inform further research in mindfulness-based intervention in people with dementia, to accelerate development of the third wave psychotherapy to extend its benefit in this disadvantaged population.

\section{Studies reviewed}

\subsection{Literature search}

We conducted a systematic literature search on the available evidence using the PsycINFO, EMBASE, MEDLINE, Web of Science, CINAHL Plus and the Cochrane Library databases. Search terms specified the population $\left(\operatorname{cog}^{*}\right.$ impair ${ }^{*}$ OR traumatic brain inj* $^{*}$ OR dementia OR Alzheimer, brain damage OR head injur*) and interventions (mindfulness OR MBCT OR MBSR OR meditation) being examined. All database searches were stored in Mendeley, a reference management software. The first three authors (blinded for peer review) screened and reviewed all papers. In cases of doubt, the second last and last authors (blinded for peer review) were consulted to reach consensus. Reference lists of potential articles were reviewed to identify further additional papers. Studies on MBSR or MBCT interventions, or modifications of these programmes published in peer reviewed journals in English were included. We included studies with (1) quantitative outcome measures (psychological, cognitive and/or behavioural outcomes), and (2) a randomised 
controlled trial or a pre- and post-test design. Non-randomised studies were included as the number of randomised controlled trials was anticipated to be limited.

\subsection{Study quality}

To critically assess the methodological quality of included studies, we used a modified version of the Downs and Black's checklist [40]. As the Downs and Black checklist does not distinguish items relating to quality of the study and reporting quality of study, the strengths and weaknesses (e.g. risk of bias) of individual studies were qualitatively examined. Consistent with the York Centre for Systematic Reviews guidelines [41], information extracted included the characteristics of the study, design and methods used, number and characteristics of participants, intervention details, outcomes and results. Where data were missing from the published studies, attempts were made to obtain this through correspondence with the trial authors. Data were extracted from all included papers. Meta-analyses were not possible due to the heterogeneous nature of the study outcomes in the papers included in this review. The review findings are presented in narrative form, according to the type of clinical presentation.

\subsection{Included studies}

In people with cognitive impairment other than dementia, 12 papers representing 10 studies were identified, including five studies in people with traumatic brain injury [42-47], two in stroke [48, 49], two in a mixed population of stroke and traumatic brain injury $[50,51]$, and one in $\mathrm{MCl}[52,53]$. In people with dementia, two studies were identified $[54,55]$. Table 1 summarises key characteristics and findings of the 
studies in cognitive impairments other than dementia. Methodological quality varied greatly (Downs \& Black's total score range, 7-21/27; Table 2).

\section{Mindfulness-based intervention in people with cognitive impairments other}

\section{than dementia: current scenario}

\subsection{Intervention protocol modifications}

Most studies have considered modifications in the intervention protocol to accommodate for the cognitive impairments (e.g., attention) and other physical needs (e.g., visual impairment) in these populations. In MBCT developed for people with acquired brain injuries such as stroke and traumatic brain injuries, several studies have shortened the session duration from the original 120 minutes to between 90 and 105 minutes, and addressed cognitive difficulties in these populations by adapting the delivery of intervention, such as increase in repetition, use of simplified language and visual aids, and shortened meditation sessions [43, 44, 48]. One shortened the home practice duration to 20-30 minutes [43]. In one study, an additional psychoeducation session on stroke was included, as well as simplified yoga movements to accommodate physical difficulties, and meeting participants individually to review their home practice and log entries [48].

In MBSR, modifications for this population include the introduction of brief mindfulness training as "attentional control training" to participants [42], omitting the retreat session $[49,50]$, and taught an additional meditation technique called lovingkindness [49], reducing the duration per session from the original 150 minutes per session to between 45 and 120 minutes [46], and increasing the total number of sessions from eight to between 10-12 sessions [45-47]. One study kept the home practice duration at 45 minutes [50]. Two studies explicitly mentioned that 
modifications were made to address the cognitive challenges in the $A B I$ population. To address the difficulties in learning, memory and fatigue, Johansson et al [50] allowed more time for participants to reflect in sessions. Azulay et al [46] made a number of modifications including: shortened session duration, increased number of sessions, reduced group size to allow time to explain and repeat concepts, provided written information of home practice, increased modelling of abstract concepts (e.g., using mindfulness to explore emotional and physical pain), asking participants to record the home practice frequency and making observations regarding their experiences.

Interestingly, in the study of people with $\mathrm{MCl}[52,53]$, no modification was made with the MBSR protocol, except for a shorter programme duration of 120 minutes. This study nevertheless reported a high attendance rate, no dropout, and no adverse events, suggesting that MBSR with little modification may be feasible in people with $\mathrm{MCl}$.

In terms of facilitator, three studies used two facilitators per group [43, 46, 48] and three studies reported using one facilitator [42, 44, 51]. Bédard et al [43] specifically trained the facilitators to teach MBCT to the participants with cognitive impairment (see training details of the facilitators in [56]).

\subsection{Outcomes}

Depressive symptoms and quality of life are the key outcomes in most randomised controlled trials of mindfulness-based interventions in people with acquired brain injuries [43-45, 48, 49], mostly using measurements commonly used in other 
populations, such as the Beck Depression Inventory-II (BDI-II)[57] and Patient Health Questionnaire (PHQ-9)[58]. Significant reduction in overall depression is observed in three MBCT studies [43, 44, 48] and one MBSR study [49] using BDI-II, and in the cognitive-affective domain of BDI-Il in one MBSR study [45]. A study on brief mindfulness training for attentional problems did not find any significant effects of the intervention on depressive symptoms [42], measured using Hospital Anxiety and Depression Scale (HADS) [59]. In studies with longer-term follow-up design, maintained reduction in depressive symptoms at 3 months was observed in two MBCT studies [43, 48], and at 1 year in an MBSR study on the cognitive-affective domain of BDI-II [47]. Improvements in both general and health-related quality of life have been reported, in both MBCT $[43,44,48]$ and MBSR $[45,46]$ studies.

The potential benefits of mindfulness-based intervention on cognitive rehabilitation are another reason for the interest in their application in people with non-developmental cognitive deficits. Cognitive tests measuring attention, learning and memory, working memory and executive functioning have been used. One study found significant improvements in sustained attention and working memory after MBSR [46], although similar results were not observed after a brief mindfulness training [42]. Johansson et al found improved information processing speed on Trail Making Test A after MBSR [50], and improved attention on an attentional blink task [51].

In the $\mathrm{MCl}$ study $[52,53]$, a whole range of measurements were used, including psychosocial measures of depression using the Center of Epidemiologic Studies Depression Scale (CESD), Life Orientation Test-Revised (LOT-R), 
Perceived Stress Scale (PSS), Quality of Life-Alzheimer's Disease (QoL-AD), Resilience Scale, Herth Hope Index, and Mindfulness Attention Awareness Scale (MAAS); a battery of cognitive outcomes including the Alzheimer's Disease Assessment Scale cognitive subscale (ADAS-Cog), Animal Naming, Boston Naming, Rey Auditory Verbal Learning Test (RAVLT), Controlled Oral Word Association Test (COWAT), and the Trail Making Test A \& B; and neurobiological measures using MRI. The studies however did not find any significant effects in these psychological/psychosocial outcomes, except that the controls performed better than the MBSR group on tests of executive functioning (i.e., Trail-Making Test Parts A and $B$ ). The authors suggested this unexpected result was due to the order of testing and fatigue [52]. On the other hand, the study has shown initial evidence of benefits in neurobiological measures using MRI, namely an increased functional connectivity in nodes in the default mode network, and trends of less hippocampal atrophy with MBSR [53].

\subsection{Implications for studies in dementia}

While people with chronic cognitive deficits are a heterogenous group, the evidence from other acquired neurological and brain conditions have important reference values for dementia. First, both MBCT and MBSR appear feasible in people with cognitive impairments, with only minor modifications required, mainly in delivery details such as duration and number of sessions, without having to tailor the intervention content according to cognitive ability (e.g., abstract thinking). This is in line with a study showing that a dementia diagnosis may not undermine a person's ability in mindful attention [60], based on a comparison of performance between people with dementia and those without on a measure of mindful attention to the 
breath; there were also no correlation between performance on this task and executive function or overall cognition.

Given that MBCT and MBSR protocols can be applied without drastic modifications based on cognitive function, it is possible that the benefits of these interventions observed in other populations can be expected in people with dementia, although empirical evidence is needed, as there are also important differences in factors influencing the key outcome domains (e.g., depression, quality of life, cognition) in this population. For example, well-being in people with dementia often shows close relationship with that of the carer, and interventions in the context of a dyadic relationship are suggested, with some authors proposing mindfulnessbased interventions for couples to enhance resilience and autonomy in handling future challenges [61].

\section{Mindfulness-based interventions in dementia: evidence review}

\subsection{Intervention protocol modifications}

In the earlier study done by Lantz et al [54], people with moderate to severe dementia were recruited in a care home; the more recent study by Litherland \& Robertson [55] was conducted in people living in their own accommodation, although the severity of dementia was not specified. Lantz and colleagues [54] modified the programme considerably to accommodate the needs of the participants with dementia at the nursing home. Participants were grouped according to the severity of dementia (mild and moderate-severe) and intervention content (modified meditation, guided imagery, and auditory, olfactory, tactile and motor body awareness). This study emphasized the importance of the facilitator modifying the 
intervention to suit the participants' interests and needs, invited nursing home staff to attend the treatment group as participants and recommended using two facilitators: one to demonstrate techniques and another to direct participants who were agitated, restless or in need of additional assistance. On the other hand, Litherland \& Robertson modified the intervention delivery only slightly, by delivering a taster session (60-90 minutes), and shortening the length of formal meditation practices and the duration of home practice [55].

\subsection{Outcomes}

In Lantz and colleagues' study in nursing home using a non-randomised controlled design, significant reduction in agitation was reported by staff in the treatment group [54]. Although the study described grouping participants according to dementia severity, it was not clear whether treatment response differed in people with different severity level. In the community sample study using a single-group pretest-postest design, no improvement was observed in overall mental health with MBSR [55]. The authors suggested that since the aim of mindfulness was to increase participants' awareness and understanding of their mental wellbeing, this might account for the lack of improvements in the short term.

\subsection{What conclusions can we draw?}

Despite the great needs, feasibility, and potential benefits as suggested in other cognitive deficit populations, empirical studies of mindfulness-based intervention using/adapting evidence-based protocols of MBCT or MBSR in people with dementia is seriously lacking. Due to the limited number of studies, varied methodological quality (lack of control group in one study, unclear risk of bias due to lack of 
adequate reporting in certain domains, such as allocation concealment, and blinding of staff to group allocation), and the small sample sizes, it is unclear whether mindfulness-based interventions may benefit people with dementia. External validity for the two included studies were poor due to recruitment difficulties, using convenience sampling, and limited details regarding the source population and intervention setting. It was also not possible to evaluate long-term effects of mindfulness-based interventions due to the limited duration of follow-up measurements. Other methodological issues include a lack of intention-to-treat analysis despite high attrition rates. External validity for all studies was poor due to recruitment difficulties and convenience sampling used, and limited details regarding the source population and intervention setting. There were also confounding factors that were not considered in the statistical analysis, such as other concurrent psychotherapies, use of psychotropic medication, and the inclusion of mixed severity of dementia. All of these clearly point to an urgent need for better quality future research.

Across the spectrum of populations with non-developmental cognitive deficit, most studies used a modified version of mindfulness-based intervention to address the cognitive difficulties of this population. Common themes included: shortened meditation sessions and/or shortened duration of each session, memory aids, increased repetition, simplified language, and omitting the retreat session. The components of the mindfulness-based interventions such as length, duration and the settings and outcome measures used varied across studies, leading to differences in intensity and "dosage" of the interventions. This further contributed to difficulties in interpreting the data. Interestingly, the study that modified the intervention the least 
had no dropout [52], although it should be cautioned that people with $\mathrm{MCl}$ are subclinical in terms of their cognitive deficits, and the need for modification to address cognitive difficulties are presumably less in this group.

Also of note is the outcome measures used in the identified studies in dementia. These studies used a diverse range of outcome measures, which made direct comparison difficult. In the earlier study in nursing home residents, agitation measured using the Cohen-Mansfield Agitation Inventory (CMAI) [62] was the only quantitative outcomes reported [54]. In the community sample of people with dementia, a generic measure of mental wellbeing, the Warwick-Edinburgh Mental Well-being Scale (WEMWBS) [63], was used. These measurement choices highlighted difference in intervention objectives (e.g., behavioural and psychological symptom control versus mental wellbeing) and considerations in selecting the most relevant outcomes in view of diagnosis, illness severity (e.g., the influence of cognitive ability in response pattern on WEMWBS [64]), and assessment burden affecting outcomes given their impaired attention.

\section{Expert opinion}

There is currently insufficient empirical evidence to draw any firm conclusion on whether mindfulness-based interventions are beneficial for people with dementia. Based on evidence from populations with cognitive impairment other than dementia, it appears that MBCT and MBSR protocols are feasible without requiring drastic modifications based on cognitive function. There is anecdotal evidence of benefit (in reducing agitation) in moderate to severe dementia; the possibility of mindfulness- 
based intervention being effective across different stages of dementia cannot be ruled out.

Mindfulness-based interventions are complex and their evaluation is methodologically challenging [65]. This challenge is further complicated by the heterogeneous nature of dementia and $\mathrm{MCl}$, and the needs for tailoring and adaptation [66], with most modifications not specified using a manual. As noted in systematic reviews on mindfulness-based interventions in other populations such as intellectual disability [67], it is not clear how closely the reviewed mindfulness-based programmes in people with dementia or $\mathrm{MCl}$ conformed to mindfulness principles. The development of manuals would enhance the replicability of future studies, allowing examination of treatment compliance or treatment fidelity. For example, in a feasibility pilot study, a 10-sesion group-based adapted mindfulness programme for people with mild to moderate dementia in care homes has been manualised, allowing evaluation on the feasibility of recruitment, retention, attrition and acceptability, and estimates on the quality-of-life benefits to inform a fully powered randomised controlled trial [68].

As highlighted in this review, the challenges in the scientific study of this complex intervention in a complex disorder have resulted in a near nonexistence of empirical evidence in an area of potentially high impact. The two studies in people with dementia that have used an evidence-based protocol of mindfulness-based intervention identified in this review were conducted across a period of 17 years [54, 55], both having a low methodology score. To advance our knowledge and practice, further research with high methodological quality, sufficiently power, rigorous design such as randomised controlled trials with longer follow-up period are urgently 
needed. It is only when measurement artefact and methodological flaws can be ruled out, more solid conclusions can be made regarding the clinical benefits of mindfulness-based interventions in dementia and $\mathrm{MCl}$.

There is also a need to study additional standardised and validated outcomes. In particular, to inform the theoretical basis of the application of mindfulness-based interventions in dementia and $\mathrm{MCl}$, measures of mindfulness should be included to assess whether the intervention's effectiveness was due to an increase in mindfulness skills or other nonspecific, latent factors. Future research using a mixedmethods approach may be beneficial, with qualitative components of studies providing important supplementary information, such as their perception, acceptability, and preference on the modifications made in the intervention.

Given the chronic nature of cognitive impairments and its impact on the person's learning curve, a slower pace with more frequent home practice appear to be needed in the intervention design. Future studies can require participants to record the frequency of practice, and explore whether increase in home practice correlates with increase in mindfulness and/or improvements in symptoms, functioning, and wellbeing. As it might take longer than the standard eight weeks for participants to embed mindfulness into their lives, a longer follow-up period would help to reflect this.

Finally, although not reviewed here, the use of mindfulness-based interventions in dementia carers have been evaluated in many studies [69-71]. The potential of intervention designs that involve the carer is being explored, including a 
10-session group-based programme for people with mild to moderate dementia, with an aim to empower people with dementia in managing psychological distress with support from carers [72], and interventions provided to dyads of people with dementia and their carers [73].

\section{Funding}

This paper was not funded.

\section{Declaration of interest}

The authors have no relevant affiliations or financial involvement with any organization or entity with a financial interest in or financial conflict with the subject matter or materials discussed in the manuscript. This includes employment, consultancies, honoraria, stock ownership or options, expert testimony, grants or patents received or pending, or royalties.

\section{Reviewer disclosures}

Peer reviewers on this manuscript have no relevant financial or other relationships to disclose.

\section{References}

Papers of special note have been highlighted as either of interest $\left(^{*}\right)$ or of considerable interest $\left({ }^{* *}\right)$ to readers.

1. Prince M, Comas-Herrera A, Knapp M, et al. World Alzheimer Report 2016: Improving Healthcare for Poeple Living with Dementia. London: Alzheimer's Disease International; 2016. ${ }^{*}$

A comprehensive report by the Alzhimer's Disease International reviewing the state of healthcare for dementia globally

2. Alzheimer's Disease International. Policy Brief for Heads of Government: The

Global Impact of Dementia 2013-2050. London: Alzheimer's Disease International; 2013. 
3. Cummings JL, Morstorf T, Zhong K. Alzheimer's disease drug-development pipeline: few candidates, frequent failures. Alzheimers Res Ther. 2014;6:37. doi: $10.1186 / a l z r t 269$

4. Wong G, Knapp M. Should we move dementia research funding from a cure to its care? Expert Rev Neurother. 2020 Apr;20:303-5. doi: $10.1080 / 14737175.2020 .1735364$

5. Prince M, Bryce R, Ferri C, et al. World Alzheimer Report 2011: The Benefits of Early Diagnosis and Intervention. London: Alzheimer's Disease International; 2011.

6. Spector A, Thorgrimsen L, Woods B, et al. Efficacy of an evidence-based cognitive stimulation therapy programme for people with dementia: randomised controlled trial. Br J Psychiatry. 2003;183:248-54.

7. National Institute for Health and Care Excellence. Dementia: A NICE-SCIE Guideline on Supporting People With Dementia and Their Carers in Health and Social Care (Revised). Leicester: National Institute for Health and Clinical Excellence; 2011

8. Woods B, Aguirre E, Spector AE, et al. Cognitive stimulation to improve cognitive functioning in people with dementia. Cochrane Database Syst Rev. 2012;2:CD005562. doi: 10.1002/14651858.CD005562.pub2

9. Huntley JD, Gould RL, Liu K, et al. Do cognitive interventions improve general cognition in dementia? A meta-analysis and meta-regression. BMJ Open. 2015;5:e005247. doi: 10.1136/bmjopen-2014-005247

10. National Institute for Health and Care Excellence. Dementia: Assessment, Management and Support for People Living with Dementia and Their Carers. London: National Institute for Health and Clinical Excellence;; 2018.** 
The latest UK national guidelines on dementia which include recommendations on non-pharmacological interventions for enhancing cognition and quality of life in people with dementia and caregivers.

11. Spector A, Orrell M, Davies S, et al. Reminiscence therapy for dementia. Cochrane Database Syst Rev. 2000:CD001120. doi: 10.1002/14651858.CD001120

12. Woods B, Spector A, Jones C, et al. Reminiscence therapy for dementia. Cochrane Database Syst Rev. 2005 18:CD001120. doi: 10.1002/14651858.CD001120.pub2

13. Woods B, O'Philbin L, Farrell EM, et al. Reminiscence therapy for dementia. Cochrane Database Syst Rev. 2018;3:CD001120. doi: 10.1002/14651858.CD001120

14. Kudlicka A, Martyr A, Bahar-Fuchs A, et al. Cognitive rehabilitation for people with mild to moderate dementia. Cochrane Database Syst Rev. 2019. doi: 10.1002/14651858.CD013388

15. Romeo R, Zala D, Knapp M, et al. Improving the quality of life of care home residents with dementia: cost-effectiveness of an optimized intervention for residents with clinically significant agitation in dementia. Alzheimers Dement. 2019;15:282-91. doi: 10.1016/j.jalz.2018.08.010

16. Chenoweth L, King MT, Jeon $\mathrm{YH}$, et al. Caring for Aged Dementia Care Resident Study (CADRES) of person-centred care, dementia-care mapping, and usual care in dementia: a cluster-randomised trial. Lancet Neurol. 2009;8:317-25. doi: 10.1016/S1474-4422(09)70045-6

17. Livingston $\mathrm{G}$, Barber J, Marston L, et al. Clinical and cost-effectiveness of the Managing Agitation and Raising Quality of Life (MARQUE) intervention for 
agitation in people with dementia in care homes: a single-blind, clusterrandomised controlled trial. Lancet Psychiatry. 2019;6:293-304. doi: $10.1016 / S 2215-0366(19) 30045-8$

18. Michalowsky B, Xie F, Eichler T, et al. Cost-effectiveness of a collaborative dementia care management: results of a cluster-randomized controlled trial. Alzheimers Dement. 2019. doi: 10.1016/j.jalz.2019.05.008

19. Knapp M, Wong G. Economics and mental health: the current scenario. World Psychiatry. 2020;19:3-14. doi: 10.1002/wps.20692

20. Orgeta V, Qazi A, Spector AE, et al. Psychological treatments for depression and anxiety in dementia and mild cognitive impairment. Cochrane Database Syst Rev. 2014:CD009125. doi: 10.1002/14651858.CD009125.pub2

21. Hayes SC, Hofmann SG. The third wave of cognitive behavioral therapy and the rise of process-based care. World Psychiatry. 2017;16:245-6. doi: 10.1002/wps.20442.*

A brief commentary on concepts and methods that are central to third wave interventions, including mindfulness methods, and the relationship within the cognitive behavioral therapy tradition and of evidence-based therapy

22. Kabat-Zinn J. Full catastrophe living: using the wisdom of the body and the mind to face stress, pain and illness. London: Piatkus; 2013.

23. Segal ZJ, Williams MG, Teasdale JD. Mindfulness based cognitive therapy for depression: a new approach to preventing relapses. New York: Guildford Press; 2002.

24. Lutz A, Slagter HA, Dunne JD, et al. Attention regulation and monitoring in meditation. Trends Cogn Sci. 2008;12:163-9. doi: 10.1016/j.tics.2008.01.005

25. Sipe WE, Eisendrath SJ. Mindfulness-based cognitive therapy: theory and 
practice. Can J Psychiatry. 2012;57:63-9. doi: 10.1177/070674371205700202

26. Hofmann SG, Sawyer AT, Witt AA, et al. The effect of mindfulness-based therapy on anxiety and depression: a meta-analytic review. J Consult Clin Psychol. 2010;78:169-83. doi: 10.1037/a0018555

27. Piet J, Hougaard E. The effect of mindfulness-based cognitive therapy for prevention of relapse in recurrent major depressive disorder: a systematic review and meta-analysis. Clin Psychol Rev. 2011;31:1032-40. doi: 10.1016/j.cpr.2011.05.002

28. Chiesa A, Serretti A. Mindfulness based cognitive therapy for psychiatric disorders: a systematic review and meta-analysis. Psychiatry Res. 2011;187:441-53. doi: 10.1016/j.psychres.2010.08.011

29. Fjorback LO, Arendt M, Ornbol E, et al. Mindfulness-based stress reduction and mindfulness-based cognitive therapy: a systematic review of randomized controlled trials. Acta Psychiatr Scand. 2011;124:102-19. doi: 10.1111/j.16000447.2011.01704.x

30. National Institute for Health and Care Excellence. Depression (NICE guideline CG90). National Institute for Health and Care Excellence; 2009.

31. Chiesa A, Serretti A. A systematic review of neurobiological and clinical features of mindfulness meditations. Psychol Med 2010;40:1239-52. doi: $10.1017 / S 0033291709991747$

32. Grossman P, Niemann L, Schmidt S, et al. Mindfulness-based stress reduction and health benefits: a meta-analysis. J Psychosom Res. 2004;57:35-43. doi: 10.1016/S0022-3999(03)00573-7

33. Simpson R, Booth J, Lawrence M, et al. Mindfulness based interventions in multiple sclerosis: a systematic review. BMC Neurol. 2014;14:15. doi: 
10.1186/1471-2377-14-15

34. British Psychological Society. Guidance on the assessment and diagnosis of intellectual disabilities in adulthood. Leicester: British Psychological Society; 2015.

35. Albert MS, DeKosky ST, Dickson D, et al. The diagnosis of mild cognitive impairment due to Alzheimer's disease: recommendations from the National Institute on Aging-Alzheimer's Association workgroups on diagnostic guidelines for Alzheimer's disease. Alzheimers Dement. 2011;7:270-9. doi: 10.1016/j.jalz.2011.03.008

36. Petersen RC. Mild cognitive impairment. Continuum (Minneap Minn). 2016;22(2 Dementia):404-18. doi: 10.1212/CON.0000000000000313

37. Petersen RC, Lopez O, Armstrong MJ, et al. Practice guideline update summary: mild cognitive impairment: report of the guideline development, dissemination, and implementation subcommittee of the American Academy of Neurology. Neurology. 2018;90:126-35. doi: 10.1212/WNL.0000000000004826

38. Lawrence M, Booth J, Mercer S, et al. A systematic review of the benefits of mindfulness-based interventions following transient ischemic attack and stroke. Int J Stroke. 2013;8:465-74. doi: 10.1111/ijs.12135

39. Ulrichsen KM, Kaufmann T, Dorum ES, et al. Clinical utility of mindfulness training in the treatment of fatigue after stroke, traumatic brain injury and multiple sclerosis: a systematic literature review and meta-analysis. Front Psychol. 2016;7:912. doi: 10.3389/fpsyg.2016.00912*

An earlier review providing initial evidence of mindfulness-based interventions applied in neurological conditions that affect cognition, focusing on fatigue. 
40. Downs $\mathrm{SH}$, Black $\mathrm{N}$. The feasibility of creating a checklist for the assessment of the methodological quality both of randomised and non-randomised studies of health care interventions. J Epidemiol Community Health. 1998;52:377-84. doi: 10.1136/jech.52.6.377

41. University of York. Systematic reviews: Centre for Reviews and Dissemination's guidance for undertaking reviews in health care 2009. Available from http://www.york.ac.uk/inst/crd/pdf/Systematic_Reviews.pdf.

42. McMillan $\mathrm{T}$, Robertson $\mathrm{IH}$, Brock $\mathrm{D}$, et al. Brief mindfulness training for attentional problems after traumatic brain injury: a randomised control treatment trial. Neuropsychol Rehabil. 2002;12:117-25.

43. Bedard M, Felteau M, Marshall S, et al. Mindfulness-based cognitive therapy reduces symptoms of depression in people with a traumatic brain injury: results from a randomized controlled trial. J Head Trauma Rehabil. 2014;29:E13-22. doi: 10.1097/HTR.0b013e3182a615a0

44. Bedard M, Felteau M, Marshall S, et al. Mindfulness-based cognitive therapy: benefits in reducing depression following a traumatic brain injury. Adv Mind Body Med. 2012;26:14-20.

45. Bedard M, Felteau M, Mazmanian D, et al. Pilot evaluation of a mindfulnessbased intervention to improve quality of life among individuals who sustained traumatic brain injuries. Disabil Rehabil. 2003;25:722-31. doi: $10.1080 / 0963828031000090489$

46. Azulay J, Smart CM, Mott T, et al. A pilot study examining the effect of mindfulness-based stress reduction on symptoms of chronic mild traumatic brain injury/postconcussive syndrome. J Head Trauma Rehabil. 2013;28:32331. doi: 10.1097/HTR.0b013e318250ebda 
47. Bédard M, Felteau M, Gibbons C, et al. A mindfulness-based intervention to improve quality of life among individuals who sustained traumatic brain injuries: one-year follow-up. J Cogn Rehabil. 2005;23:8-13.

48. Moustgaard A, Bédard M, Felteau M. Mindfulness-based cognitive therapy (MBCT) for individuals who had a stroke: results from a pilot study. J Cogn Rehabil 2007;25:4-10.

49. Joo HM, Lee SJ, Chung YG, et al. Effects of mindfulness based stress reduction program on depression, anxiety and stress in patients with aneurysmal subarachnoid haemorrhage. J Korean Neurosurg Soc. 2010;47:345-51.

50. Johansson B, Bjuhr $\mathrm{H}$, Ronnback L. Mindfulness-based stress reduction (MBSR) improves long-term mental fatigue after stroke or traumatic brain injury. Brain Inj. 2012;26:1621-8. doi: 10.3109/02699052.2012.700082

51. Johansson B, Bjuhr H, Karlsson M, et al. Mindfulness-based stress reduction (MBSR) delivered live on the internet to individuals suffering from mental fatigue after an acquired brain injury. Mindfulness. 2015;6:1356-65.

52. Wells RE, Kerr CE, Wolkin J, et al. Meditation for adults with mild cognitive impairment: a pilot randomized trial. J Am Geriatr Soc. 2013;61:642-5. doi: $10.1111 /$ jgs.12179

53. Wells RE, Yeh GY, Kerr CE, et al. Meditation's impact on default mode network and hippocampus in mild cognitive impairment: a pilot study. Neurosci Lett. 2013 ;556:15-9. doi: 10.1016/j.neulet.2013.10.001

54. Lantz MS, Buchalter EN, McBee L. The Wellness Group: a novel intervention for coping with disruptive behavior among elderly nursing home residents. Gerontologist. 1997;37:551-6. doi: 10.1093/geront/37.4.551 
55. Litherland R, Robertson G. Mindfulness meditation: can it make a difference? J Dement Care. 2014;22:31-3.

56. Gibbons C, Felteau M, Cullen N, et al. Training clinicians to deliver a mindfulness intervention. Mindfulness. 2014;5:232-7.

57. Steer RA, Ball R, Ranieri WF, et al. Dimensions of the Beck Depression Inventory-II in clinically depressed outpatients. J Clin Psychol. 1999;55:117-28. doi: 10.1002/(sici)1097-4679(199901)55:1<117::aid-jclp12>3.0.co;2-a

58. Kroenke K, Spitzer RL, Williams JB. The PHQ-9: validity of a brief depression severity measure. J Gen Intern Med. 2001;16:606-13. doi: 10.1046/j.15251497.2001.016009606.x

59. Zigmond AS, Snaith RP. The hospital anxiety and depression scale. Acta Psychiatr Scand. 1983;67:361-70. doi: 10.1111/j.1600-0447.1983.tb09716.x

60. Bousfield C, Stott J. Impact of dementia on mindful attention: a crosssectional comparison of people with dementia and those without. Mindfulness. 2019;10:279-87. doi: https://doi.org/10.1007/s12671-018-0972-6**

A recent study investigating whether people with dementia perform worse on mindful attention, and whether mindful attention is related to executive function and overall cognition in this population.

61. Berk L, Warmenhoven F, van Os J, et al. Mindfulness training for people with dementia and their caregivers: rationale, current research, and future directions. Front Psychol. 2018;9:982. doi: 10.3389/fpsyg.2018.00982** A review on explaining the rationale for applying mindfulness in dementia care, which includes an overview of on mindfulness for people with dementia.

62. Cohen-Mansfield J, Marx MS, Werner P. Agitation in elderly persons: an integrative report of findings in a nursing home. Int Psychogeriatr. 1992;4 
Suppl 2:221-40. doi: 10.1017/s1041610292001285

63. Tennant R, Hiller L, Fishwick R, et al. The Warwick-Edinburgh Mental Wellbeing Scale (WEMWBS): development and UK validation. Health Qual Life Outcomes. 2007;5:63. doi: 10.1186/1477-7525-5-63

64. Deary IJ, Watson R, Booth $\mathrm{T}$, et al. Does cognitive ability influence responses to the Warwick-Edinburgh Mental Well-Being Scale? Psychol Assess. 2013;25:313-8. doi: 10.1037/a0030834

65. Moore GF, Audrey S, Barker M, et al. Process evaluation of complex interventions: Medical Research Council guidance. BMJ. 2015;350:h1258. doi: 10.1136/bmj.h1258

66. Craig P, Dieppe P, Macintyre S, et al. Developing and evaluating complex interventions: the new Medical Research Council guidance. BMJ. 2008;337:a1655. doi: 10.1136/bmj.a1655

67. Chapman MJ, Hare DJ, Caton S, et al. The use of mindfulness with people with intellectual disabilities: a systematic review and narrative analysis. Mindfulness. 2013;4:179-89.

68. Churcher Clarke A, Chan JMY, et al. An adapted mindfulness intervention for people with dementia in care homes: feasibility pilot study. Int J Geriatr Psychiatry. 2017;32:e123-e131. doi: 10.1002/gps.4669

69. Brown KW, Coogle CL, Wegelin J. A pilot randomized controlled trial of mindfulness-based stress reduction for caregivers of family members with dementia. Aging Ment Health. 2016;20:1157-1166. doi: 10.1080/13607863.2015.1065790

70. Hou RJ, Wong SY, Yip BH, et al. The effects of mindfulness-based stress reduction program on the mental health of family caregivers: a randomized 
controlled trial. Psychother Psychosom. 2014;83:45-53. doi: $10.1159 / 000353278$

71. Whitebird RR, Kreitzer M, Crain AL, et al. Mindfulness-based stress reduction for family caregivers: a randomized controlled trial. Gerontologist. 2013;53:676-86. doi: 10.1093/geront/gns126

72. Chan J, Churcher Clarke A, Royan L, et al. A Mindfulness Program Manual for People With Dementia. Behav Modif. 2017;41:764-87. doi: $10.1177 / 0145445517715872$

73. Berk L, Warmenhoven F, Stiekema APM, et al. Mindfulness-based intervention for people with dementia and their partners: results of a mixedmethods study. Front Aging Neurosci. 2019;11:92. doi: 10.3389/fnagi.2019.00092 


\section{Legends}

Table 1. Description of studies reviewed

Table 2. Quality scores for studies included using a modified version of the Downs \& Black's (1998) checklist 
Table 1. Description of studies reviewed

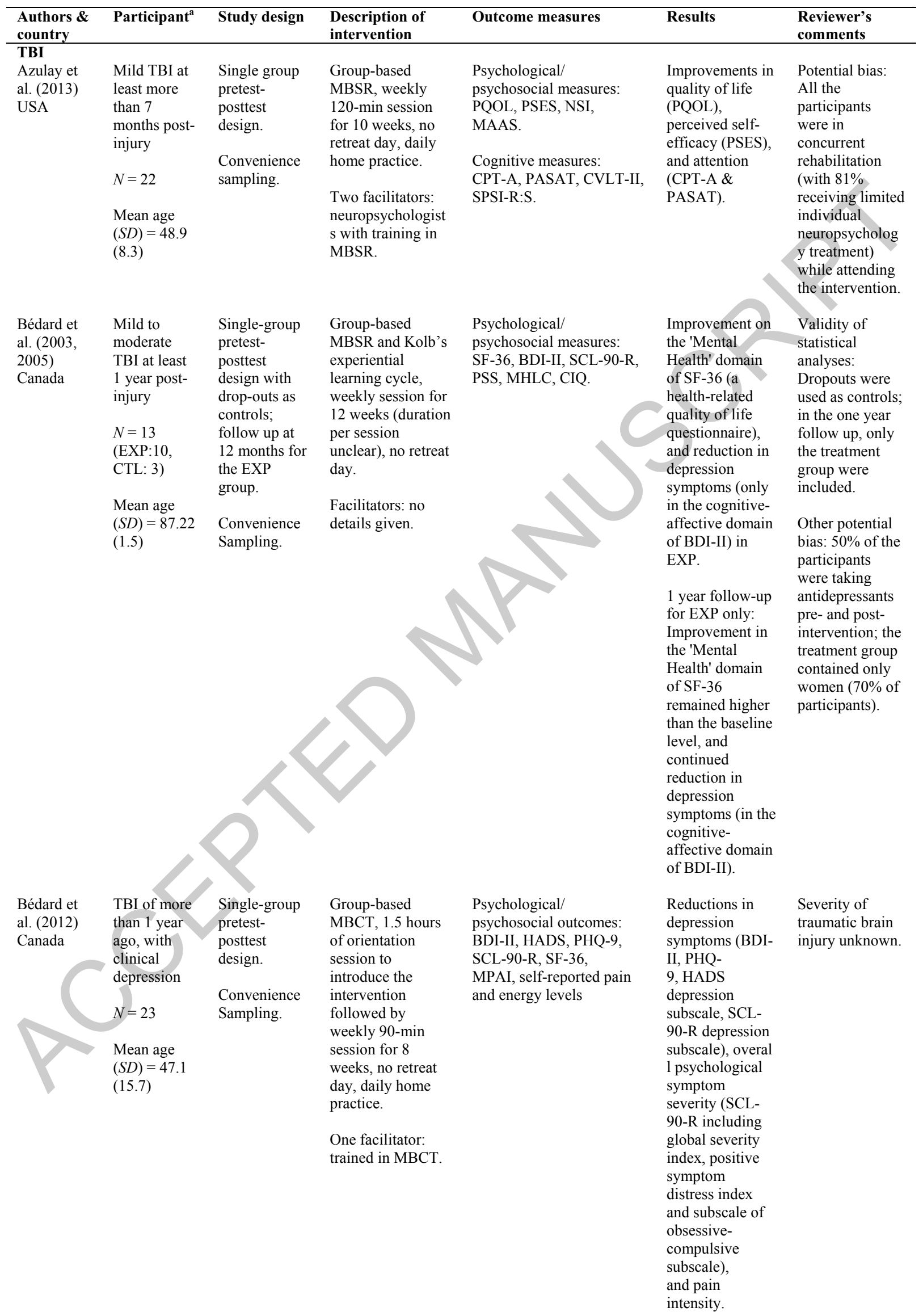




\begin{tabular}{|c|c|c|c|c|c|c|}
\hline $\begin{array}{l}\text { McMillan } \\
\text { et al. } \\
(2002) \\
\text { UK }\end{array}$ & $\begin{array}{l}\text { TBI of } \\
\text { mixed } \\
\text { severity } \\
\text { between } 3- \\
12 \text { months } \\
\text { post-injury } \\
N=145 \\
\text { (EXP: } 50 \text {; } \\
\text { PE: } 47 \text {; } \\
\text { CTL:48) } \\
\text { Mean age } \\
\text { (SD) } \\
\text { EXP: } 34.6 \\
\text { (11.4) } \\
\text { PE: } 31.4 \\
(13.0) \\
\text { CTL:36.2 } \\
\text { (13.4) }\end{array}$ & $\begin{array}{l}\text { Pretest- } \\
\text { posttest RCT; } \\
\text { follow up at } 6 \\
\text { months and } \\
12 \text { months } \\
3 \text { groups: } \\
\text { EXP, physical } \\
\text { exercise (PE) } \\
\text { and CTL. }\end{array}$ & $\begin{array}{l}\text { 1:1 ACT (only } \\
\text { mindfulness } \\
\text { breathing } \\
\text { technique in } \\
\text { MBSR was } \\
\text { taught), } 45 \text {-min, } 5 \\
\text { sessions in } 4 \\
\text { weeks, no retreat } \\
\text { day, home } \\
\text { practice. } \\
\text { One facilitator: } \\
\text { therapist with no } \\
\text { mindfulness-based } \\
\text { training (no further } \\
\text { details given). }\end{array}$ & $\begin{array}{l}\text { Psychological outcomes: } \\
\text { HADS, GHQ, and } \\
\text { Rivermead Post- } \\
\text { Concussional Symptoms } \\
\text { Questionnaire } \\
\text { Cognitive outcomes: } \\
\text { TEA, AMIPB, PASAT, } \\
\text { TMT Trails A \& B, EMQ } \\
\text { and CFQ }\end{array}$ & $\begin{array}{l}\text { No significant } \\
\text { findings for all } \\
\text { three groups post } \\
\text { intervention, and } \\
\text { at 6- and 12- } \\
\text { month follow- } \\
\text { ups. }\end{array}$ & $\begin{array}{l}\text { The intervention } \\
\text { only included } \\
\text { teaching one } \\
\text { MBSR } \\
\text { technique on an } \\
\text { individual basis } \\
\text { at participant's } \\
\text { home. }\end{array}$ \\
\hline $\begin{array}{l}\text { Bédard et } \\
\text { al. (2014) } \\
\text { Canada }\end{array}$ & 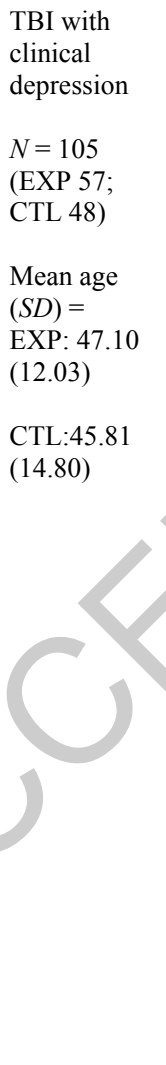 & $\begin{array}{l}\text { Pretest- } \\
\text { posttest } \\
\text { randomised } \\
\text { wait-list } \\
\text { controlled } \\
\text { trial; follow } \\
\text { up at } 3 \\
\text { months. } \\
\text { Stratified } \\
\text { randomisation } \\
\text { of sample to } \\
\text { ensure } \\
\text { balance } \\
\text { between } \\
\text { groups on } \\
\text { symptoms of } \\
\text { depression } \\
\text { (using BDI-II } \\
\text { score), age } \\
\text { and sex. }\end{array}$ & $\begin{array}{l}\text { Group-based } \\
\text { MBCT, weekly } \\
90 \text {-min session for } \\
10 \text { weeks, no } \\
\text { retreat day, daily } \\
\text { 20-30-min } \\
\text { meditation home } \\
\text { practice. } \\
\text { Two facilitators: } \\
\text { healthcare } \\
\text { professionals } \\
\text { working in } \\
\text { rehabilitation for } \\
\text { people with } \\
\text { neurological } \\
\text { conditions trained } \\
\text { to deliver the } \\
\text { modified MBCT. }\end{array}$ & $\begin{array}{l}\text { Psychological outcomes: } \\
\text { BDI-II, PHQ-9, SCL-90-R, } \\
\text { PHLMS, TMS (for only } \\
\text { EXP). }\end{array}$ & $\begin{array}{l}\text { Reduction in } \\
\text { depression } \\
\text { symptoms in } \\
\text { EXP (BDI-II). } \\
\text { 3-month follow } \\
\text { up: } \\
\text { Reduction in } \\
\text { depression } \\
\text { symptoms was } \\
\text { maintained } \\
\text { (BDI-II). }\end{array}$ & $\begin{array}{l}\text { Risk of selection } \\
\text { bias: } 5 \text { were } \\
\text { assigned to the } \\
\text { intervention } \\
\text { without } \\
\text { randomisation. } \\
\text { Risk of attrition } \\
\text { bias: } 29 \% \\
\text { dropouts in both } \\
\text { treatment and } \\
\text { control groups } \\
\text { post } \\
\text { intervention, } \\
\text { with additional } \\
16 \% \text { and } 27 \% \\
\text { dropouts in } \\
\text { treatment and } \\
\text { control groups } \\
\text { respectively at } \\
3 \text {-month follow- } \\
\text { up. } \\
\text { Other potential } \\
\text { bias: About a } \\
\text { third of } \\
\text { participants in } \\
\text { both treatment } \\
\text { and control } \\
\text { groups had } \\
\text { antidepressant } \\
\text { medications at } \\
\text { baseline. }\end{array}$ \\
\hline $\begin{array}{l}\text { Stroke } \\
\text { Joo et al. } \\
(2010) \\
\text { Korea }\end{array}$ & $\begin{array}{l}\text { 6-months } \\
\text { post surgery } \\
\text { for } \\
\text { aneurysmal } \\
\text { subarachnoi } \\
\text { d } \\
\text { haemorrhage }\end{array}$ & $\begin{array}{l}\text { Single group } \\
\text { pretest- } \\
\text { posttest } \\
\text { design. }\end{array}$ & $\begin{array}{l}\text { Group-based } \\
\text { MBSR and loving- } \\
\text { kindness } \\
\text { meditation, weekly } \\
150 \text {-min session } \\
\text { for } 8 \text { weeks, no } \\
\text { retreat day, }\end{array}$ & $\begin{array}{l}\text { Psychological outcomes: } \\
\text { BDI-Korean version, STAI }\end{array}$ & $\begin{array}{l}\text { Reduction in } \\
\text { depression } \\
\text { symptoms (BDI- } \\
\text { Korean version). }\end{array}$ & $\begin{array}{l}\text { Risk of attrition } \\
\text { bias: } 60 \% \text { non- } \\
\text { completers; no } \\
\text { reasons for the } \\
\text { dropouts were } \\
\text { provided. }\end{array}$ \\
\hline
\end{tabular}


$N=28$

Mean age

(range) $=$

52.5 (38 to

65)

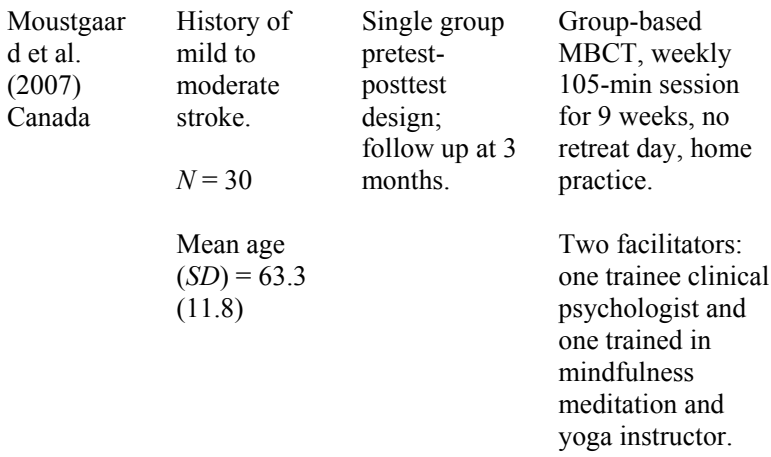

practice was

given.

Facilitators: no

details given. unclear if home

Psychological/psychosocia

1 outcomes:

BAI, BDI-II, HADS, SF-

36, SS-QoL

Improvements

post

intervention, and

Risk of attrition

bias: $23 \%$ non-

month follow up

for anxiety (BAI,

HADS),

depression (BDI-

II, HADS),

physical \&

mental health

status (SF-36),

and overall

quality of life

(SS-QoL).

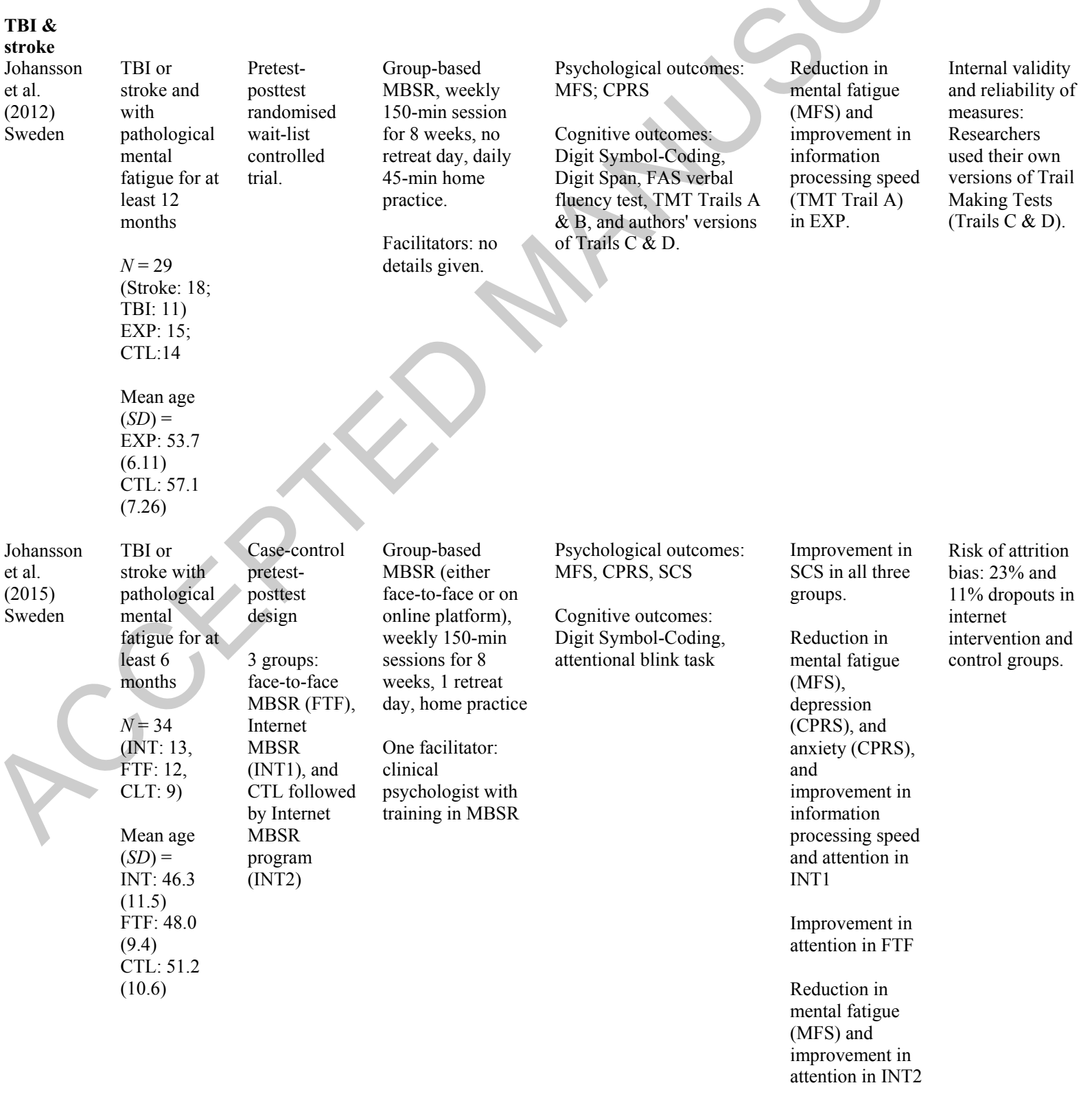




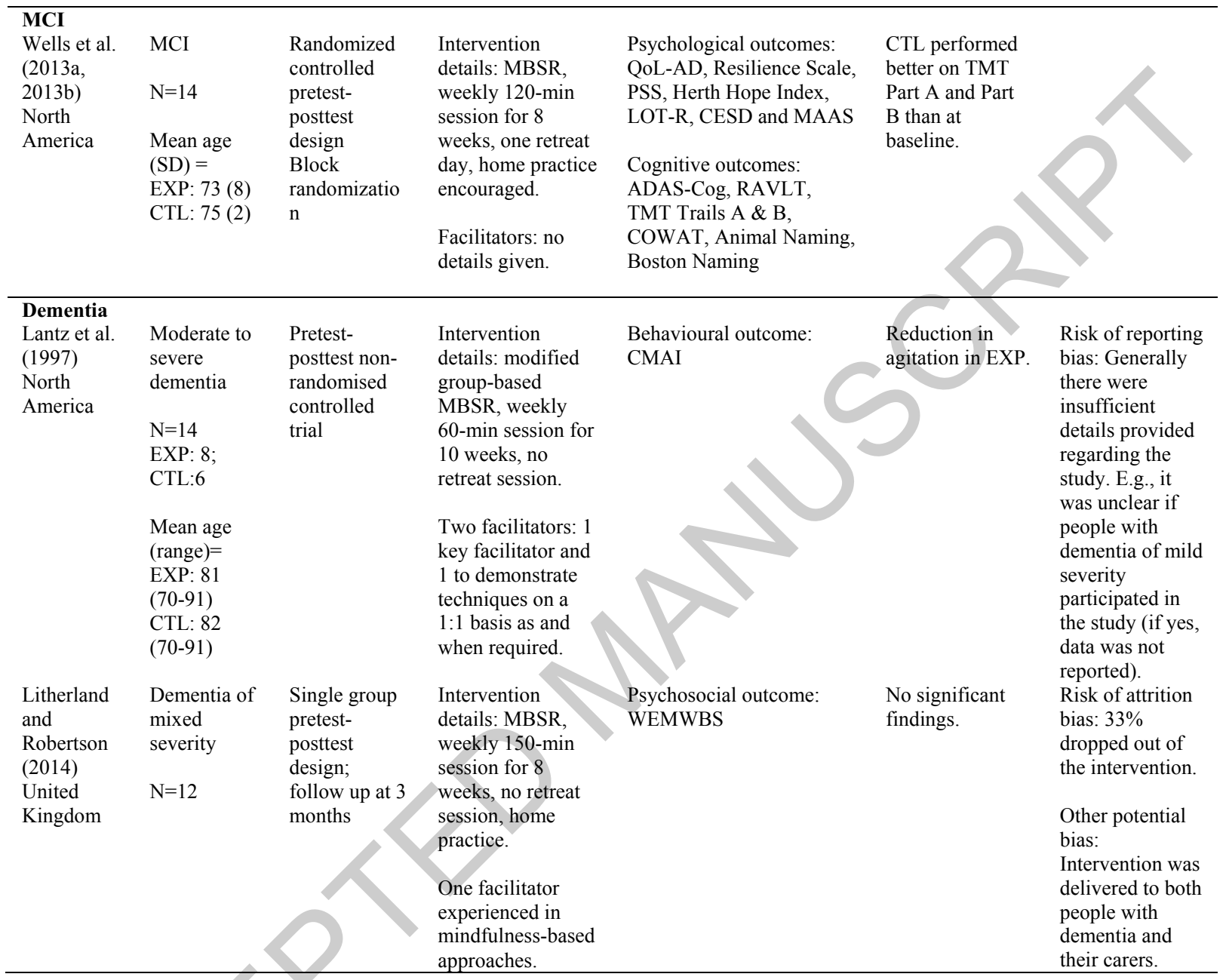

a The sample size at the start of the study, before any participants dropped out.

* All findings reported in this table were statistically significant at $\mathrm{p}<.05$.

ADAS-Cog=Alzheimer's Disease Assessment Scale cognitive subscale, ACT=Attentional Control Training, AMIPB=Adult Memory and Information Processing Battery, BDI-II $=$ Beck Depression Inventory, $\mathrm{CFQ}=\mathrm{Cognitive}$ Failures Questionnaire, $\mathrm{CESD}=\mathrm{Center}$ of Epidemiologic Studies Depression Scale, CIQ=Community Integration Questionnaire, CMAI=Cohen-Mansfield Agitation Inventory, COWAT $=$ Controlled Oral Word Association Test, CPRS = Comprehensive Psychopathological Rating Scale, CPT-A= Continuous Performance Test of Attention, CTL= Control group, CVLT-II= California Verbal Learning Test-II, EMQ=Everyday Memory Questionnaire, EXP $=$ Experimental group, $\mathrm{GHQ}=$ General Health Questionnaire, HADS=Hospital Anxiety and Depression Scale, LOT$\mathrm{R}=$ Life Orientation Test-Revised, MAAS=Mindfulness Attention Awareness Scale, $\mathrm{MCI}=$ mild cognitive impairments, MFS $=$ Mental Fatigue Scale, MHLC=Multidimensional Health Locus of Control Scale, MPAI=Mayo-Portland Adaptability Inventory, NSI= Neurobehavioural Symptom Inventory, PASAT= Paced Auditory Serial Addition Test, PHLMS=Philadelphia Mindfulness Scale, PHQ9=Patient Health Questionnaire, PQOL = Perceived Quality of Life scale, PSES $=$ Perceived Self-Efficacy Scale, PSS $=$ Perceived Stress Scale, QoL-AD=Quality of Life-Alzheimer's Disease, RAVLT=Rey Auditory Verbal Learning Test, SCL-90-R=Symptom Checklist, SCS=Self-Compassion Scale short form, SF-36=Short Form Health Survey, SPSI-R:S=Social Problem-Solving Inventory-Revised: Short form, SS QoL=Stroke Specific Quality of Life, STAI: State-Trait Anxiety Inventory, TBI=Traumatic brain injury, TEA=Test of Everyday Attention, TMS=Toronto Mindfulness Scale, TMT=Trail Making Test, WEMWBS=Warwick Edinburgh Mental Well-being Scale 
Table 2. Quality scores for studies included using a modified version of the Downs \& Black's (1998) checklist ${ }^{a}$

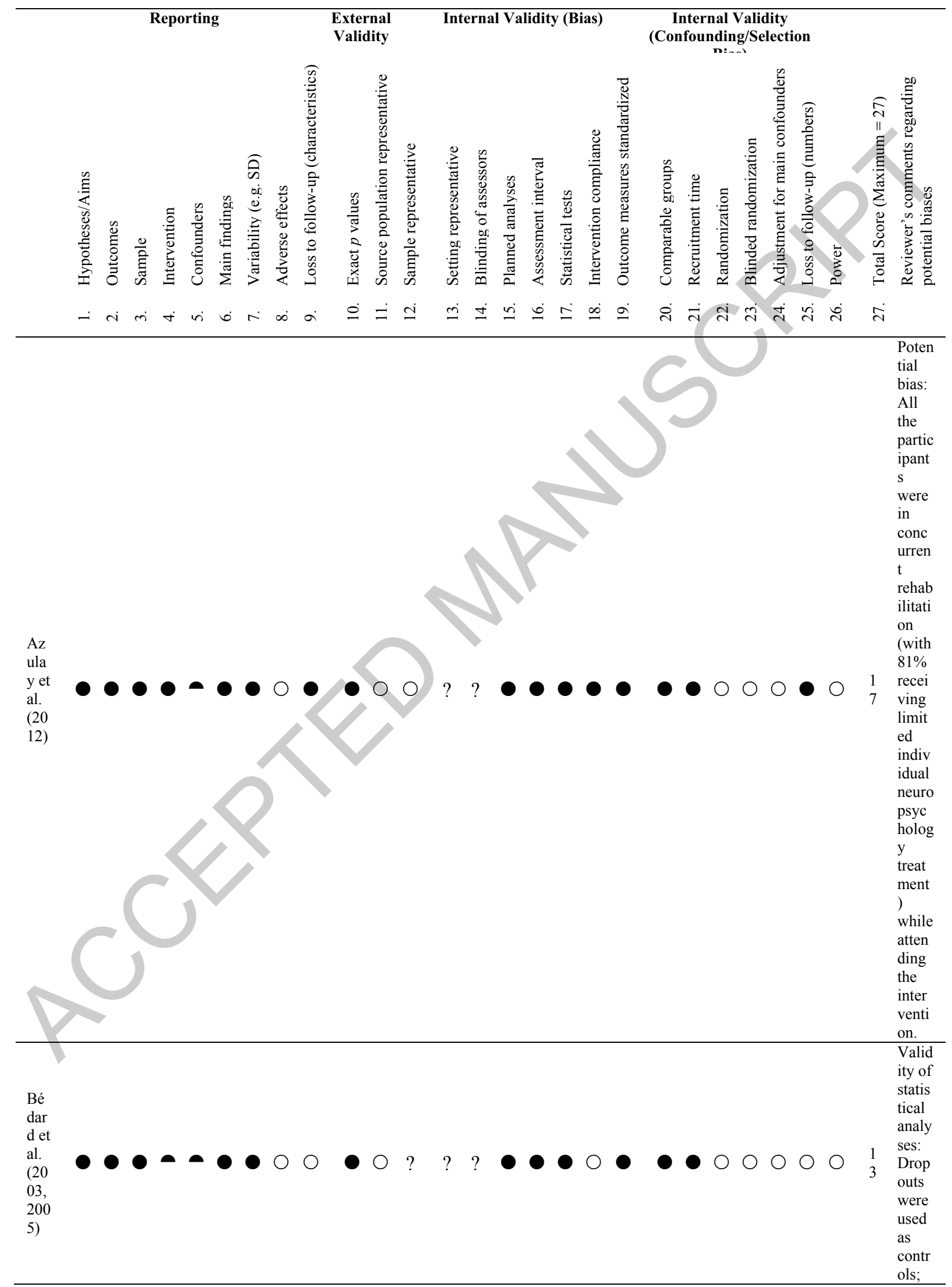




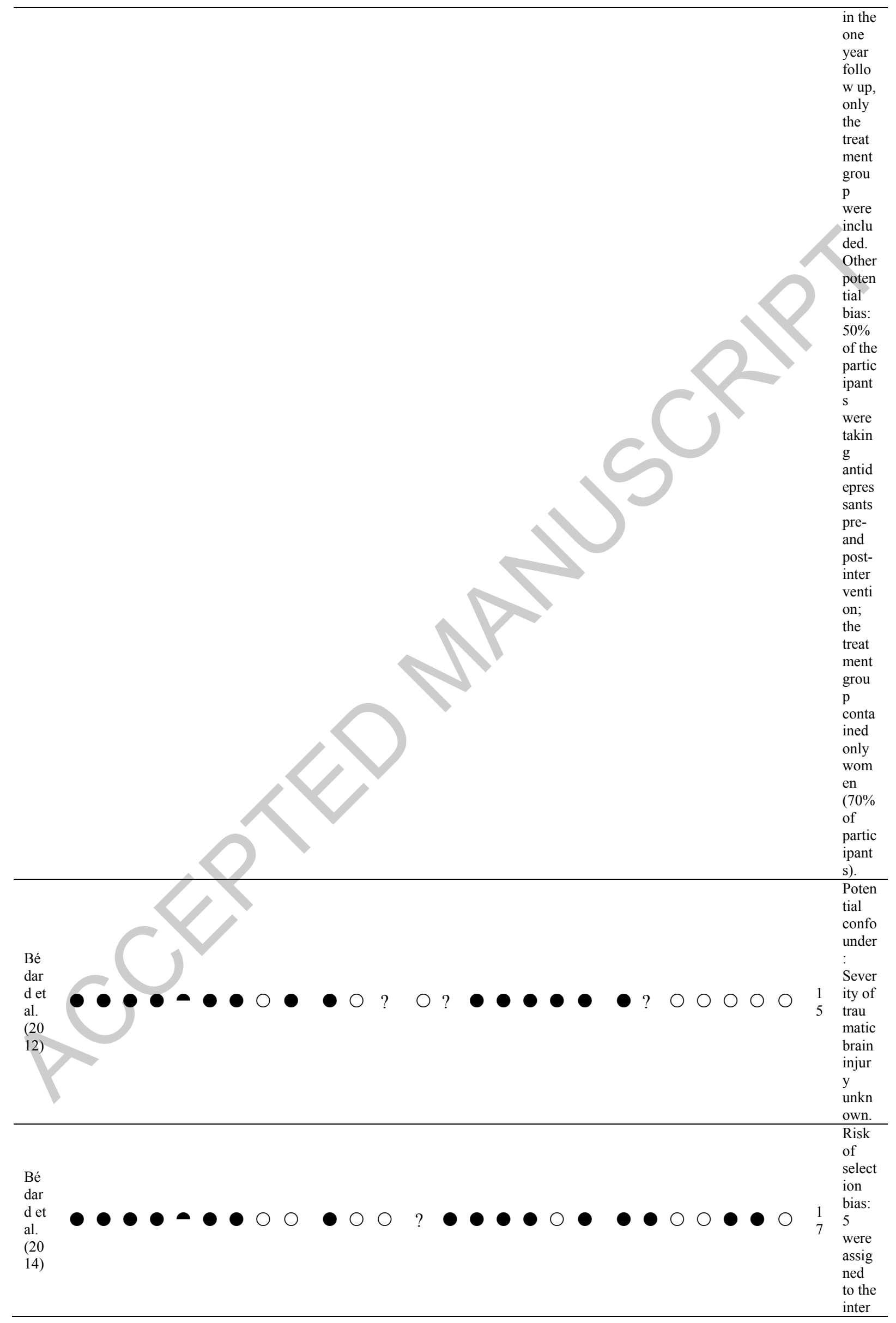




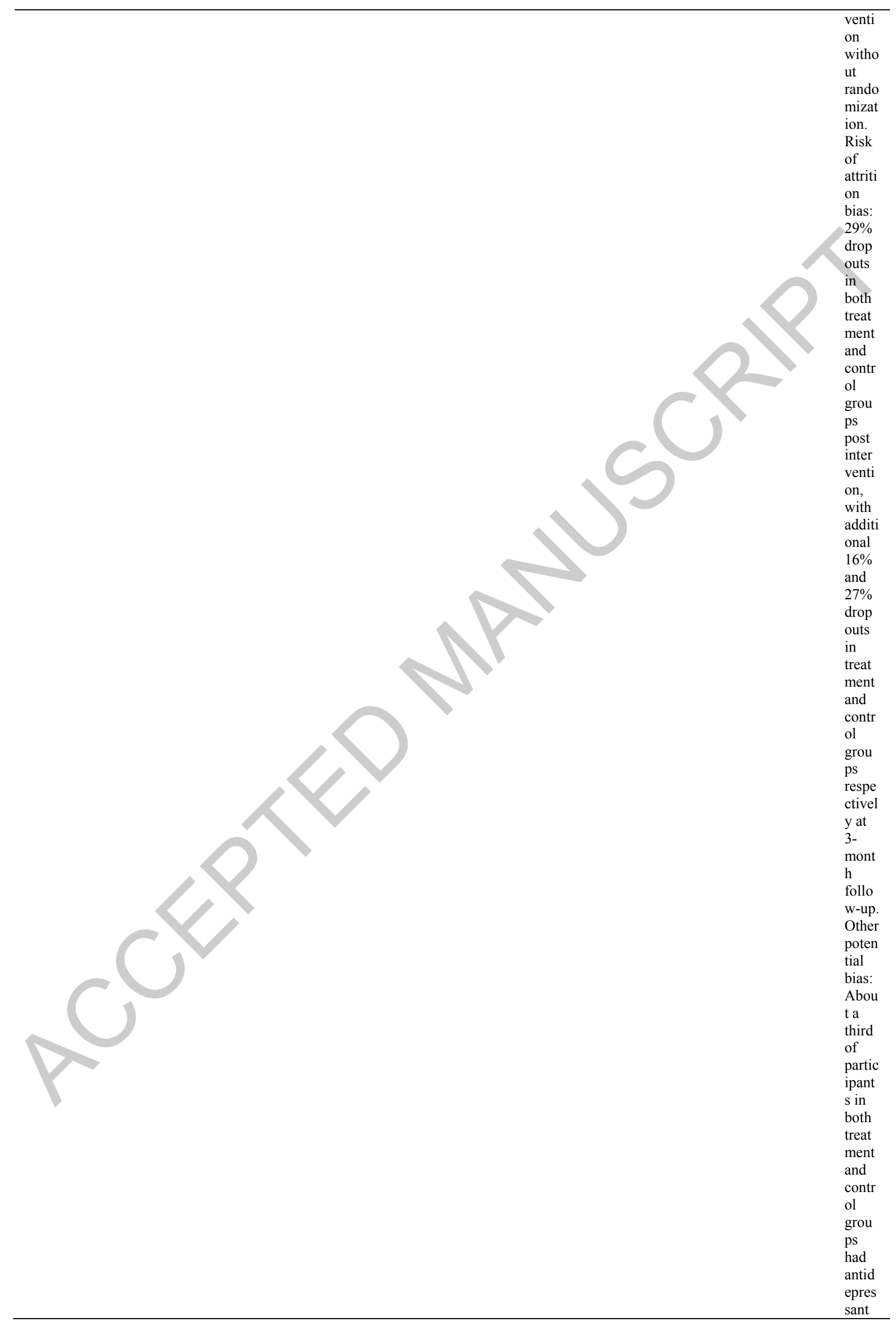




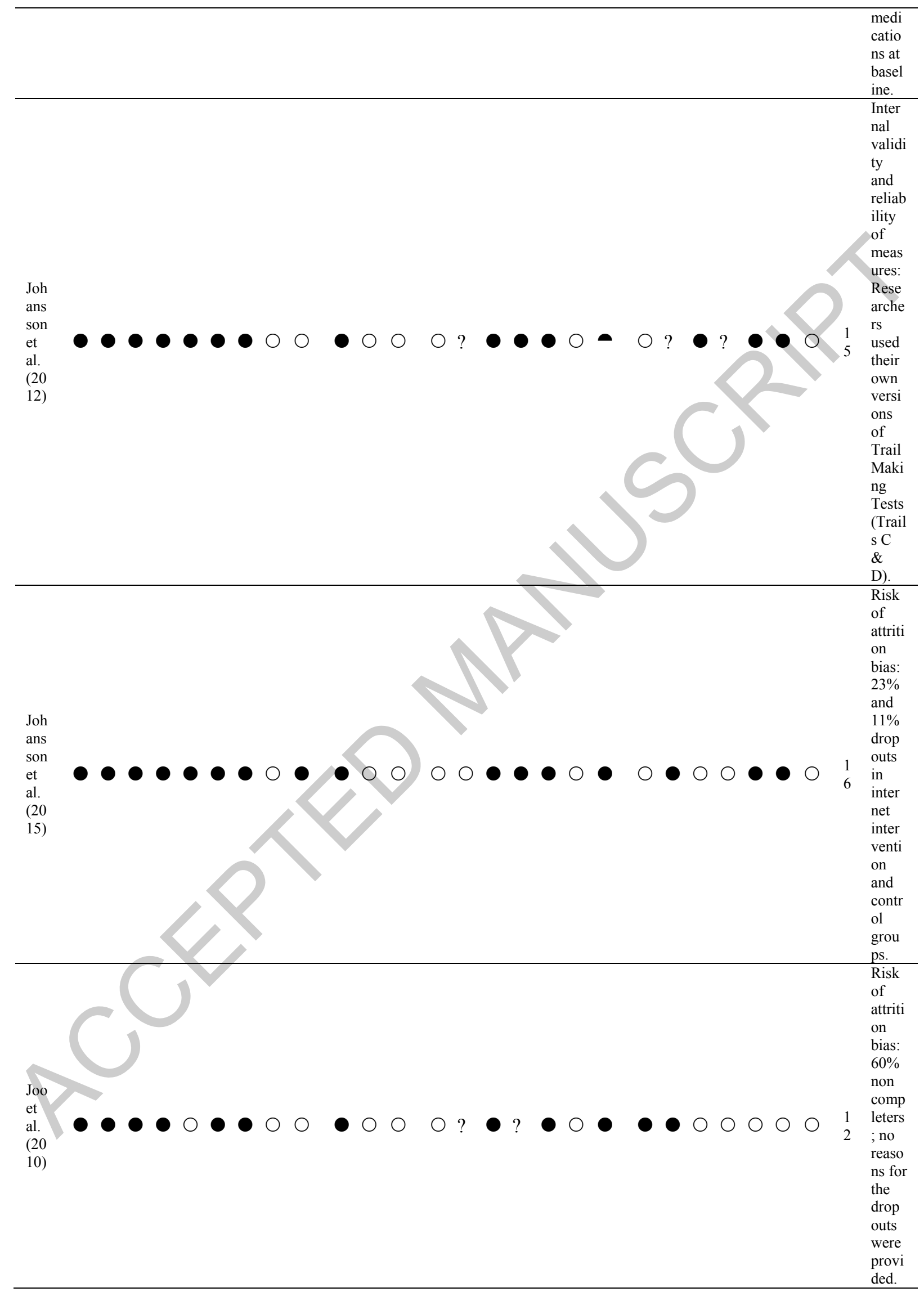




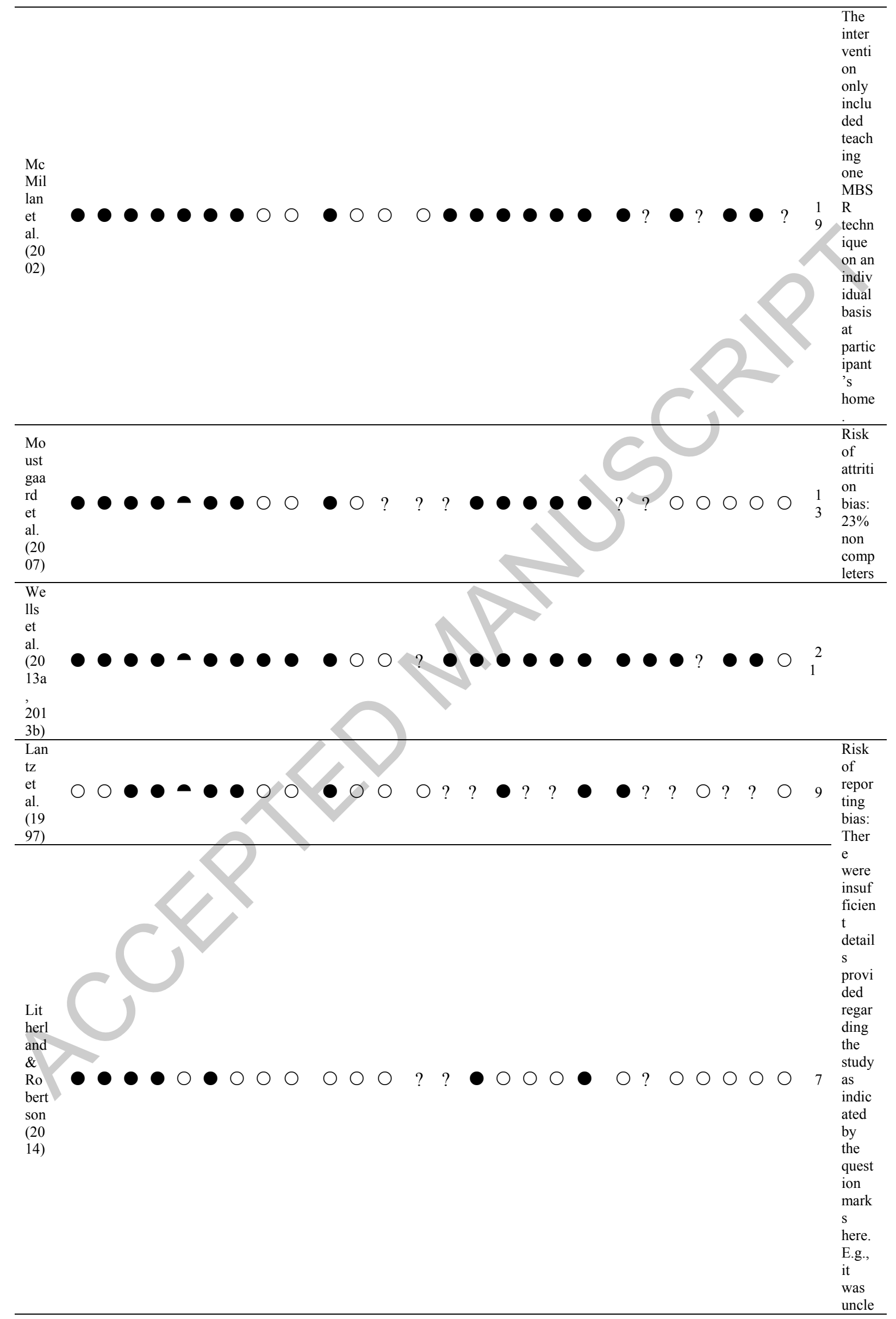




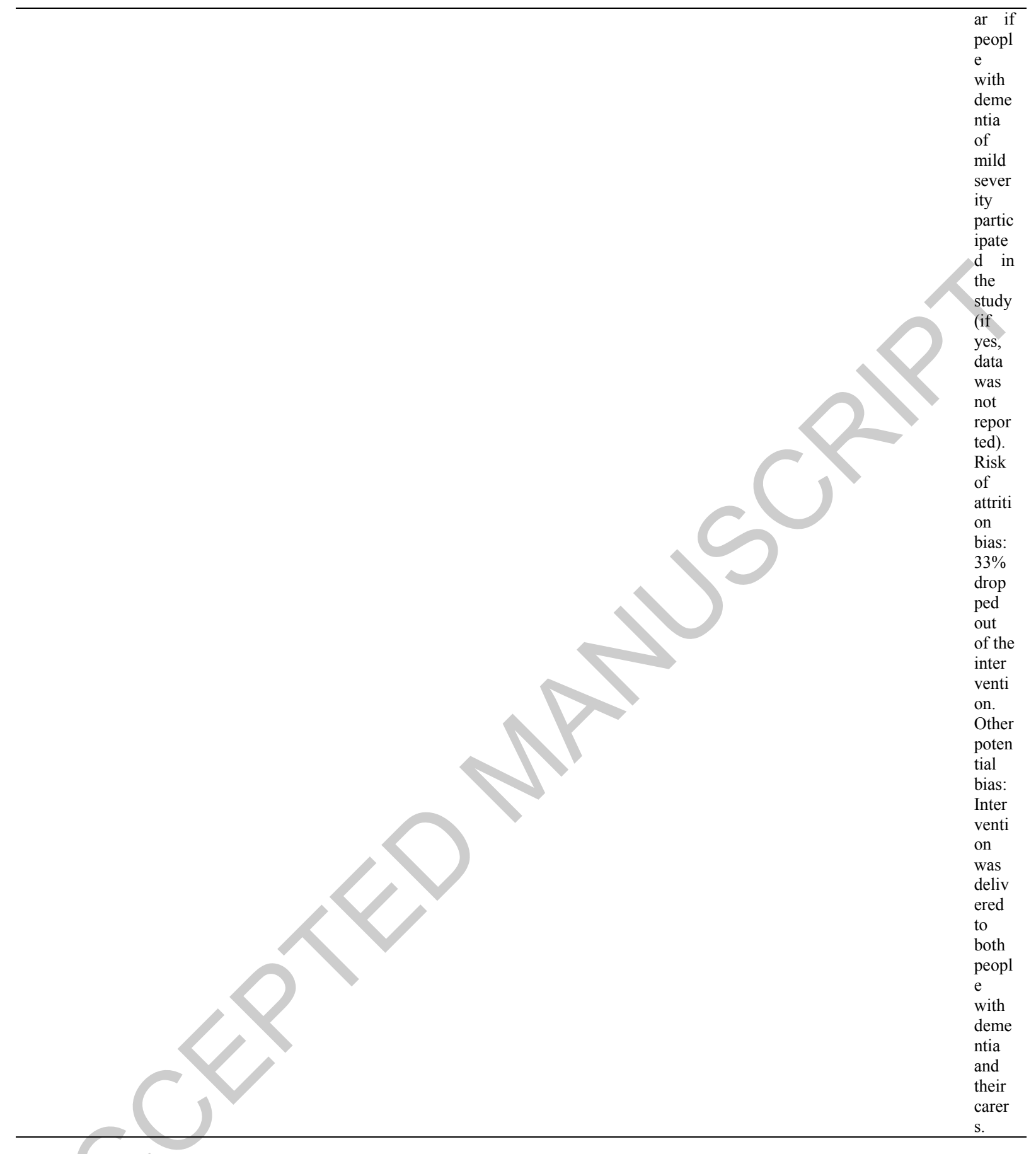

Note: - present (score of 1, or 2 for item 5); - present, with some limitations (score of 1 for item 5 or score of 0 on other items); $\bigcirc$ not present (score of 0 ); ? unable to determine (score of 0 )

${ }^{a}$ The checklist was slightly modified with item 14 removed, as blinding study subjects is not applicable for most psychological intervention studies. Item 27 asked to rate the statistical power from a score of 0 to 5 . Given that most included studies did not provide detailed power calculations, we simplified this rating to a score of 0 to 1 , to represent whether studies were or were not indicated as sufficiently powered. The total score for the modified checklist ranges between 0 and 27. 